\title{
Two-Photon Spectroscopy of Dipole-Forbidden Transitions
}

\section{Dipole-Forbidden Transitions and Double Excited Configurations in the CNDO- CI Methods}

\author{
Bernhard Dick and Georg Hohlneicher \\ Lehrstuhl für Theoretische Chemie der Universität zu Köln, D-5000 Köln, Federal Republic \\ of Germany
}

We have investigated the applicability of CNDO/S-type methods for the calculation of optical spectra of molecules with the special implication that the calculations should not only describe the intense, dipole-allowed transitions which dominate the one-photon absorption spectrum but also those transitions which are one-photon forbidden in first-order approximation. We show that such a method is well suited to predict dipole allowed and dipole forbidden transitions at a similar level of accuracy if double excited configurations are taken into account. In spite of the lack of perfect pairing in NDO methods there are still two types of states which exhibit a different sensitivity towards correlation effects. Therefore, the approximation by which we describe the $R$-dependence of the Coulomb repulsion gains much more importance than in cases where mainly dipole allowed transitions are of interest. These findings confirm results obtained earlier from theories for which the pairing theorem is valid. The calculated data show an excellent stability with respect to further increase of the number of configurations if at least about 200 energy selected configurations are taken into account.

Key words: Dipole-forbidden transitions - Two-photon spectroscopy

\section{Introduction}

For many years a great amount of experimental information on excited electronic states of conjugated $\pi$-systems has been derived from conventional UV spectroscopy based on one-photon absorption. However, this information is restricted essentially to excited states which can be reached from the ground state or from the lowest triplet state (in triplet-triplet absorption [1]) by a dipole allowed transition (DAT). In one-photon absorption a dipole forbidden transition (DFT) is usually accessible 
to direct spectroscopic investigation only in cases where the first electronic excitation is dipole forbidden and well separated from higher excitations. DFTs which lie in the region of strong absorption are seldom detectable even if they gain a considerable amount of intensity by vibronic coupling. Due to the broadening involved, this is especially true for measurements made in solution, which is often the only possible way to investigate larger systems. As a result, our experimental information on excited states is usually limited to a single part of these states. A comparable knowledge on the other part, however, is highly desirable. These states can be of great importance to other molecular properties like polarizabilities, radiationless transitions and photochemical behaviour.

A great step forward to a direct observation of DFT has been made during recent years by rapid developments in two-photon spectroscopy. At least some DFTs are usually allowed with respect to two-photon selection rules. Compared with some other experimental methods which do not depend on dipole selection rules (e.g. electron impact spectroscopy [2]), two-photon spectroscopy can be applied very well to molecules in solution [3]. Two-photon absorption spectra with good resolution are, however, still scarce and some of the information necessary for a theoretical analysis of these spectra (polarization data) is often missing. In addition, most of the assignments of two-photon absorption spectra have been based on calculations which have been shown recently to be less valid for DFT than for DAT (see Sect. 2). In this series of papers we try to provide additional theoretical and experimental information in this field.

In the first paper (I) we reinvestigate the $\mathrm{CNDO} / \mathrm{S}$ method with respect to recent developments in the general theory of electronic excitations in unsaturated molecules. We show that a properly modified $\mathrm{CNDO} / \mathrm{S}$ method is capable of yielding excitation energies which are of similar accuracy for DAT and DFT, thereby allowing a congruent discussion of all low-lying excited states.

In the second paper (II) [4], we show that the same method is very suitable for the calculation of two-photon cross-sections, quantities which are very important for the interpretation and assignment of two-photon absorption spectra.

In subsequent papers we then present a number of combined experimental and theoretical studies on different molecules, starting with some of the systems for which questions on their present assignments arise from the discussions given in I and II.

\section{Dipole Forbidden Transitions and Higher Excited Configurations}

Before we start with a brief outline of the present state of the theory of conjugated $\pi$-systems, let us define the meaning of "dipole forbidden" in this context.

In Pariser-Parr-Pople-type theories, where matrix elements of the one-electron Hamiltonian are taken into account only between nearest neighbours, occupied and unoccupied orbitals of alternant hydrocarbons are strictly "paired" [5]. This leads to a degeneracy of excited configurations which is not determined by spatial 
symmetry. In the resulting excited states these degenerate configurations always appear with the same weight but in such a way that their corresponding transition moments either add (plus-states) or subtract (minus-states) [6]. Transitions from the molecular ground state, which has to be regarded as a minus-state itself, to all other singlet minus-states have zero transition density. This is also true if the transition is allowed by symmetry [6-8].

In more advanced theories, where the one-electron matrix elements are not restricted to nearest neighbours, orbitals are no longer paired. Some of the experimental evidence seems to show that the deviations from perfect pairing are not very strong [9-11]. In connection with excited states, however, it is more likely that we have to face a "breakdown of the pairing theorem" [12]. As a consequence the transition moments for transitions into states which would be labelled "minus" in PPP approximation do no longer vanish, but in most cases they are still small. We therefore refer to a DFT as a one-photon transition

1. which is forbidden by symmetry with respect to dipole selection rules.

2. for which, though symmetry allowed, the transition moment vanishes in PPP approximation.

Let us now review some basic aspects and some recent developments in the theory of electronic spectra of conjugated $\pi$-systems.

As usual, electronic states are expanded in a basis of configurations

$$
|\psi\rangle=\sum_{\substack{i^{\prime} \cdots k^{\prime} \\ i \cdots k}} c_{i}^{i^{\prime} \cdots k^{\prime} \cdots} a_{k^{\prime}}^{+}, a_{k} \cdots a_{i}^{+}, a_{i}\left|\psi_{0}\right\rangle
$$

$a_{i}^{+}$and $a_{i}$ are one-electron creation and annihilation operators, respectively, $\left|\psi_{0}\right\rangle$ is the ground-state configuration and $c_{i}^{i^{\prime} \cdots k_{k}^{\prime}}$ are weight factors.

Due to the number of creation/annihilation operator pairs which appear in (1), the configurations can be classified in single excited configurations (SEC), double excited configurations (DEC), and so on. As usual in the treatment of conjugated $\pi$-systems, the one-electron functions which correspond to the operators $a_{i}, a_{i}^{+}$, are delocalized orbitals.

Corresponding to the approximations further involved in the derivation of quantitative treatable methods, we discern between two types of semi-empirical CI methods:

1. PPP-type methods, in which only $\pi$-electrons are treated explicitly $[5,13,14,6]$.

2. NDO-type methods, where all the valence electrons are taken into account [15-19].

For a great number of applications it is well known that these methods are very successful in the description and prediction of the optical spectra of a wide variety of unsaturated molecules, if only SECs are taken into account. Many different parametrization schemes have been proposed [5, 13-34], some of which are more suitable for special classes of molecules than others. But as a whole the average accuracy of calculated excitation energies is not altered very much if the basic 


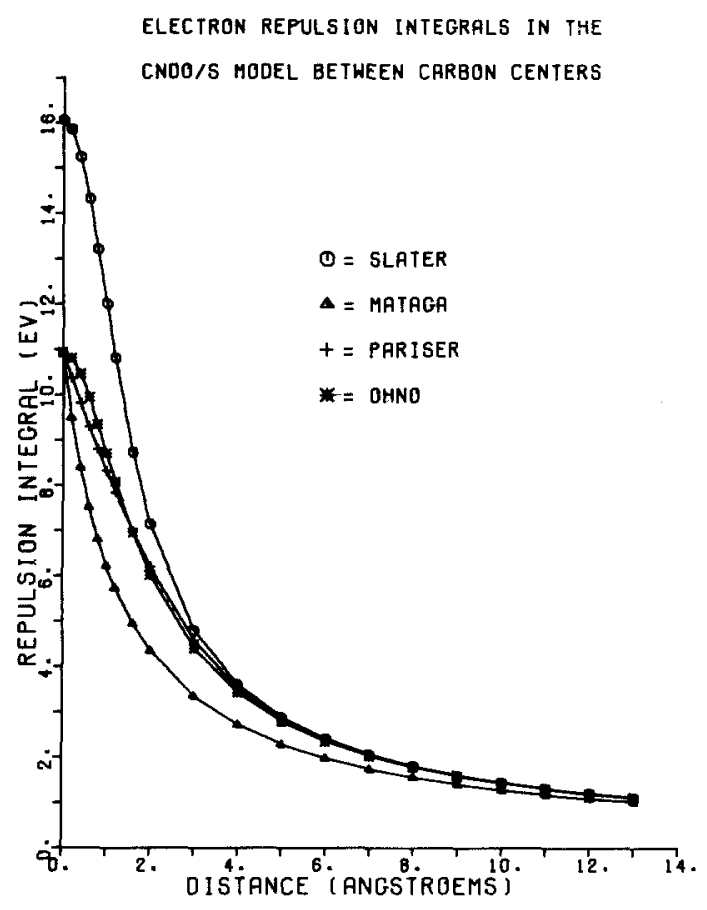

Fig. 1. Different approximations for the electron repulsion integrals as a function of internuclear distance $(R)$

parameters are varied within certain limits. This is especially true for the function by which the $R$-dependence of the effective Coulomb repulsion (ECR) between electrons located on different atoms ( $\gamma$-integrals) is described. The $\gamma$-integrals are calculated directly from Slater-orbitals [35] (S) or approximated by more or less empirical functions. The most popular of these approximations have been developed by Pariser [20] (P), Nishimoto and Mataga (NM) [21] and by Ohno and Klopman $(\mathrm{OK}){ }^{\mathrm{l}}$ These functions are shown in Fig. 1 . They mainly differ in the screening of the ECR corresponding to a different decay with increasing $R$.

In spite of the great success semi-empirical CI calculations which include only SEC (SCI) undoubtedly had in the description of optical spectra of unsaturated molecules, one should be well aware that at this stage the theory is basically a theory of dipole-allowed transitions. Due to the experimental conditions mentioned in the Introduction, most of the experimental information which has been used to adjust the parameters and to test the validity of the evaluated scheme was obtained from this type of transitions. The often drawn conclusion that a theory which is good for DAT must be good for DFT, too, is therefore not obvious. On the contrary, some facts should have previously warned against such a conclusion.

It was recognized that different parametrizations which lead to comparable results for DAT yield quite different results for the energies of low-lying triplet states [36-38,

1 This formula, which is usually referred to as Ohno's, was predicted independently by Ohno [22] and Klopman [23] at the same time. 
55]. Steeper $\gamma$-functions like MN usually give triplet energies which are too low compared with experiment.

Other hints came from purely theoretical investigations. The very early calculations of Craig et al. [39-41] in which $\gamma$-integrals calculated over Slater orbitals (S) had been used showed a strong influence on the final results of higher than single excited configurations. Later investigations [42,43], however, came to a completely different conclusion, denying a stronger influence of higher excited configurations (HEC) on the calculated energies of low-lying excitations. It was soon realized [44-46] that the relative importance of $\mathrm{HEC}$ is connected with the steepness of the $\gamma$-function. In a general analysis of this problem, Koutecký [47] has shown that the influence of correlation effects on the ordering of the low-lying excited states

1. increases with increasing steepness of the $\gamma$-function,

2. should be most pronounced in all-trans-polyenes.

However, those states which are most affected are usually not accessible from the ground state via DAT. In most cases the observable part of the optical spectrum is not changed very much by inclusion of HEC. Therefore, these results, though regarded as very interesting from the theoretical point of view, were widely neglected in practical applications and also in the more recent development of spectroscopic all-valence electron methods [16, 19].

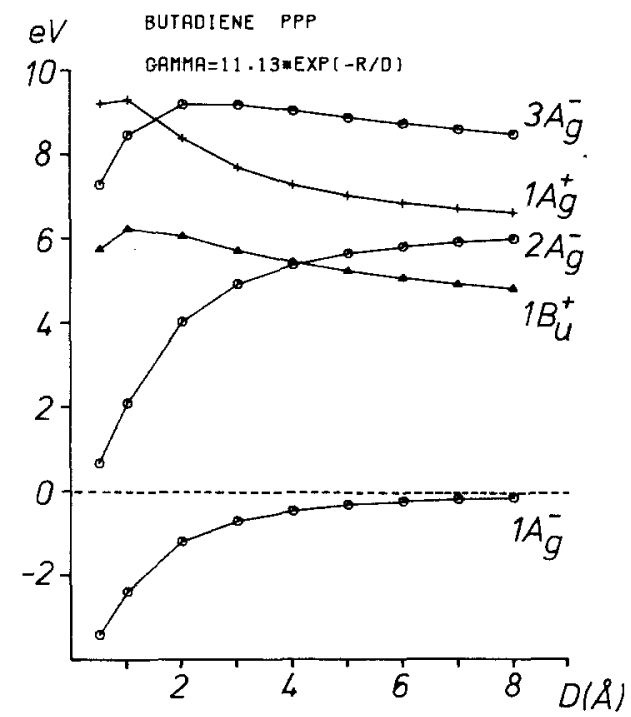

Fig. 2. Influence of the steepness of the effective Coulomb repulsion on correlation effects. Ground and excited state energies of Butadiene are shown as obtained from a PPP-type calculation including single and double excited configurations and using an exponentially decreasing model-potential. In contrast to the ionic $1 B_{u}^{(+)}$and $1 A_{g}^{(+)}$states the covalent states $1 A_{g}^{(-)}, 2 A_{g}^{(-)}$and $3 A_{g}^{(-)}$are strongly lowered in energy in the short range limit 
A strong new impact came in 1972 when Hudson and Kohler [48] and later Christensen and Kohler [49] discovered low-lying ${ }^{1} A_{g}$ states in $\alpha$, $\omega$-diphenyloctatetraene and in 2,10-dimethylundecapentaene. In SCI calculations these states lie at considerably higher energies and no feasible change in parameters allows to reproduce the experimental result. Only the inclusion of higher excited configurations yields low-lying ${ }^{1} A_{g}$ states in all-trans polyenes [50-52] in full accordance with the prediction of Koutecký [47].

Further theoretical investigations [53-55] confirmed the earlier findings. In the limit of a short-range ECR (corresponding to a steep $\gamma$-function) ${ }^{1} X^{+}$and ${ }^{3} X^{-}$ states correspond directly to ionic states in the VB description. Similarly, $1 X^{-}$and ${ }^{3} X^{+}$states correspond to covalent VB states. This distinction divides the manifold of excited states into two subsets with different physical excitation mechanisms. Only in the limit of a long-range ECR this distinction is no longer meaningful. Due to these different excitation mechanism the two subsets exhibit a different sensitivity to correlation effects, a sensitivity which strongly depends on the actual range of the ECR (Fig. 2). For a long-range interaction the influence of HEC is small and nearly uniform for all low-lying excited states; for a short-range interaction, however, covalent states show a much larger correlation effect than the ionic ones. This can lead to a complete shuffling of the excited states compared to the SCI result, but distances between states of the same subset are much less affected than those between states which belong to different subsets. Therefore, we have to keep the following in mind for all further investigations:

1. If only a theoretical description of the one-photon absorption spectrum is desired, SCI calculations should be sufficient.

2. If DAT and DFT have to be calculated at a similar level of accuracy, HEC must be included.

3. The relative importance of HEC strongly depends on the actual range of the ECR.

From quantitative calculations performed in $\pi$-approximation Schulten, Ohmine and Karplus [55] came to the conclusion that the ECR is of intermediate range, at least in polyenes and in benzene. Steep $\gamma$-functions like MN, which usually lead to very good overall results in SCI calculations, were found to over-estimate correlation effects if HEC are included. The calculations of SOK also show that there is no further shuffling of the excited states if higher than DEC are taken into account. All the low-lying excited states are shifted more or less equally, but somewhat more than the ground state. From this, one would suggest that calculations which include single and double excited configurations (SDCI) should provide a framework for the calculation of both DFT and DAT with a similar level of accuracy.

\section{Double Excited Configurations in NDO-Type Methods}

All-valence-electron methods like CNDO/S [16] or INDO/S [19], which allow the calculation of UV spectra, have gained steadily increasing interest as the investiga- 

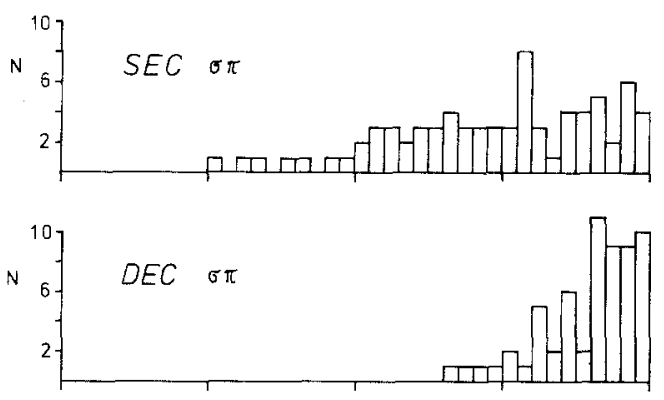

Fig. 3. Number of low-lying single and double excited configurations as function of energy. Example: Naphthalene with Pariser integrals. Upper part: overall $\sigma \pi$-symmetry. Lower part: overall $\pi \pi^{*}$ symmetry. The single excited $\sigma \rightarrow \sigma^{*}$ configurations (hatched) fall into the same energy range as the double excited $\pi \pi \rightarrow \pi^{*} \pi^{*}$ configurations
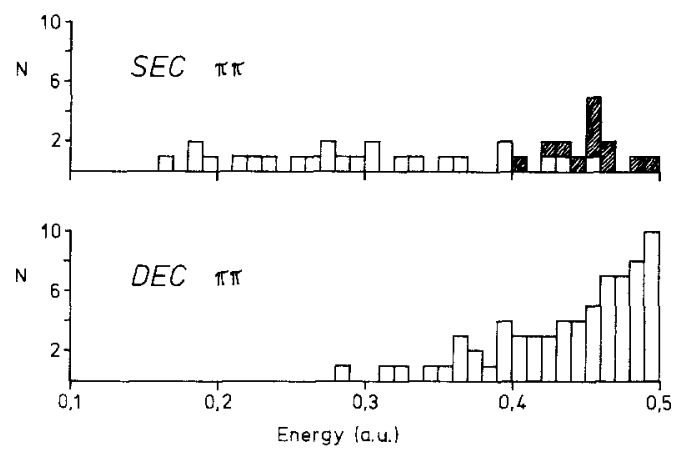

tion of non-planar $\pi$-systems, where characteristic interactions are intermediate between pure $\sigma$ and pure $\pi$, became an important field of chemical and spectroscopic research [56]. But even in the case of planar systems the use of all-valence electron methods is more appropriate if one deals with transitions of low intensity:

1. Weak bands in the UV spectrum are caused not only by DFT of overall $\pi \pi^{*}$ symmetry but also by transitions of overall $\sigma \pi$-symmetry. If these transitions originate from lone pairs they often have low excitation energies. If we try to assign the DFT which belong to the $\pi$-system it is necessary to get at least some theoretical hint on the possible appearance of low-lying $\sigma \pi$-transitions.

2. If HEC are really necessary to describe DFT properly, it is no longer possible to argue that $\sigma \sigma^{*}$-configurations are of much higher energy than the $\pi \pi^{*}$ configurations which are used to describe the low-lying transitions. From a typical example which is shown in Fig. 3, it is obvious that the lowest $\sigma \sigma^{*}$ configurations have similar energy as the lower $\pi \pi, \pi^{*} \pi^{*}$-double excitations.

Up to now no detailed information is available whether the general concepts which we have outlined in Sect. 2 also hold for methods like CNDO/S and INDO/S or not. This is by no means obvious because of the above mentioned fact that the pairing theorem is no longer valid for these methods. As an example Ellis and Jaffé [12] have shown that the degeneracies required by this theorem are not even approximated in naphthalene. As a result the plus- and minus-states no longer form two independent subsets. In consequence thereof the different sensitivity of these subsets towards correlation effects might be only an artifact of PPP theory.

The influence of HEC in CNDO-type calculations has been studied to some extent in a series of papers by Griessner-Prettre and Pullmann [57]. These authors 
Table 1. Number of possible configurations in $\pi$-and in all-valence approximation. $n=$ number of electrons treated explicitly; $\mathrm{SCI}$ : with single excited configurations; SDCI with single and double excited configurations

\begin{tabular}{lrrrlrr}
\hline & \multicolumn{2}{c}{ PPP } & \multicolumn{4}{l}{ CNDO } \\
& $n$ & SCI & SDCI & $n$ & SCI & SDCI \\
\hline Butadiene & 4 & 5 & 15 & 22 & 122 & 7503 \\
Hexatriene & 6 & 10 & 55 & 32 & 257 & 33153 \\
Benzene & 6 & 10 & 55 & 30 & 226 & 25651 \\
Octatetraene & 8 & 17 & 153 & 42 & 442 & 97903 \\
Naphthalene & 10 & 26 & 351 & 48 & 577 & 166753 \\
Anthracene & 14 & 50 & 1275 & 66 & 1090 & 594595 \\
Stilbene & 14 & 50 & 1275 & 68 & 1157 & 669903 \\
\hline
\end{tabular}

restrict their investigations to smaller molecules and they do not discuss the influence of the decay of the effective Coulomb interaction. To our opinion this is due to the fact that the importance of this question has not been recognized so clearly at that time. However, some more recent applications, where HEC are included in $\mathrm{CNDO} / \mathrm{S}$ calculations $[58,59]$ do not deal with this problem, too. It is therefore one of the main aspects of this paper to investigate to which extent the general results obtained from PPP theory can be transferred to NDO-type methods.

\section{Selection of Configurations and Stability of Results}

Before we start to investigate the influence of different $\gamma$-approximations we have to deal with another problem. If HEC are included in calculations which take into account all valence electrons and if we do not want to restrict ourselves to very small molecules, the number of possible configurations rapidly increases. For a few examples these numbers are listed in Table 1.

With computers now available, it is not difficult to perform a full SDCI calculation in $\pi$-approximation or a complete SCI calculation in the all-valence scheme for a molecule like naphthalene. A complete SDCI treatment in the latter scheme is, however, out of range. We also have to be aware that for a large number of configurations the $\mathrm{CI}$ part becomes the most time-consuming part of the calculation. It is not reasonable to perform such extensive CI calculations within the framework of a semiempirical method. The application of such methods is only justified if useful results can be obtained from calculations which do not include more than a few hundred configurations. This raises two strongly coupled questions:

1. How do we select configurations?

2. Do we obtain reasonable stability?

To the first question there exists no unique answer. Usually configurations are selected from a certain number of occupied MOs, say for example [7 $\times 7 / 3 \times 3]$, which means all SEC which evolve from the 7 highest occupied and the 7 lowest unoccupied MOs plus all DECs which evolve from the 3 highest occupied and 3 lowest unoccupied MOs. 
According to our opinion the selection of configurations should not be done in such a schematic way. It should be preferably based on the energy of configurations. However, a complete ordering of all possible configurations with respect to energy is tedious and therefore, inapt for semiempirical methods. For the succeeding calculations we have used the following procedure to select $\mathrm{N}$ configurations [60].

First we define an excitation index. This index is the number of the MOs lying between the hole and the excited electron. For DECs the excitation index is the sum of two of these distances. Within one set (SEC or DEC) the energy of configurations roughly increases with increasing excitation index. Thus, generating configurations in the order of increasing excitation index yields a presorted set. The indices for SEC and DEC are kept separately and raised alternatively, depending on the type of the two configurations which has the lower energy. From the presorted set we finally select those $N$ configurations having the smallest diagonal elements.

By application of this procedure we cannot be sure to find actually the first $N$ of all possible energy-ordered configurations. Practical application has, however, shown that provided the presorted set is not too small (a few times $N$ ) the final result is nearly unchanged if this set is increased. It is of interest to compare the result of the energy-based selection with the result of the schematic method mentioned above. In Fig. 4 such a comparison is shown for naphthalene. From the 95 configurations obtained from the schematic set $[7 \times 7 / 3 \times 3]$ only 62 belong to an energy selected set of the same length. A few SECs which evolve from the schematic set are not included in the energy selected one, but 22 SECs which do not appear in the former are included in the latter. From the 31 lowest DECs 21 belong to the $[3 \times 3]$ set but 24 from this set are not included if selection is based on energy. On the whole one finds that with respect to energy, DECs are overrepresented in the schematic set.

The second question which we have raised above is concerned with the stability of the results. For any method which is useful for a larger field of application the obtained results should not depend strongly on the point where the CI expansion is truncated. Otherwise, an arbitrary selection of this point can lead to an arbitrary result.

To study the stability of excitation properties (excitation energies, transition moments and two photon cross sections) obtained from CNDO/S-type calculations

Fig. 4. Comparison of two different procedures for the selection of configurations

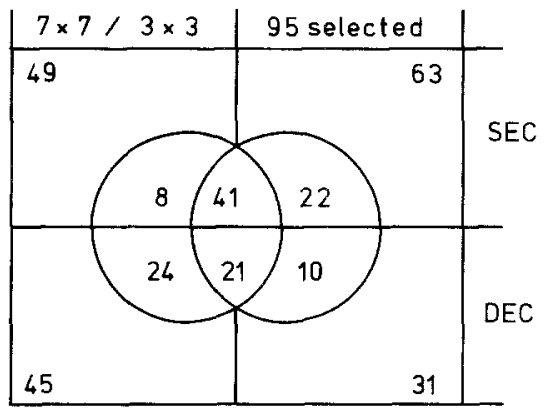


which include single and double excited configurations, we have performed three different SDCI calculations for most of the molecules discussed in Sect. 6:

1. A calculation with 200 energy selected configurations (200 t).

2. A calculation with 200 energy selected configurations of overall $\pi \pi^{*}$-symmetry $\left(200 \pi \pi^{*}\right)$.

3. A calculation with 200 energy selected configurations in each irreducible representation of the symmetry group $(200 \mathrm{e})$. This corresponds to a total number of 800 or 1600 configurations, depending on the molecule.

To give an impression of the general result obtained from these calculations we discuss two examples (benzene and hexatriene). As far as stability is concerned, the different $\gamma$-approximations basically lead to the same result. We therefore confine our discussions to only one $\gamma$-function in each case.

Let us first have a look to the evolution of calculated energies for the low-lying singlet excitations of benzene in CNDO/S (MN approximation) as the number of SECs increases (Fig. 5). For standard CNDO/S where one uses about 60 SECs the calculated energies for transitions to $1 B_{2 u}, 1 B_{1 u}$ and $1 E_{1 u}$ are in good agreement with experiment. At that point one takes into account all possible $\pi \pi^{*}$ but only $7 \%$ of the $\sigma \sigma^{*}$ configurations. If the number of configurations is further increased, the energies of the states $1 B_{1 u}, 1 E_{1 u}$ and $1 E_{2 g}$ steadily decrease, due to the interaction with $\sigma \sigma^{*}$ configurations of the same symmetry. The only state-besides the ground state - which does not change in energy with further increase of the number of configurations is $1 B_{2 u}$. This is due to the high symmetry of benzene in connection
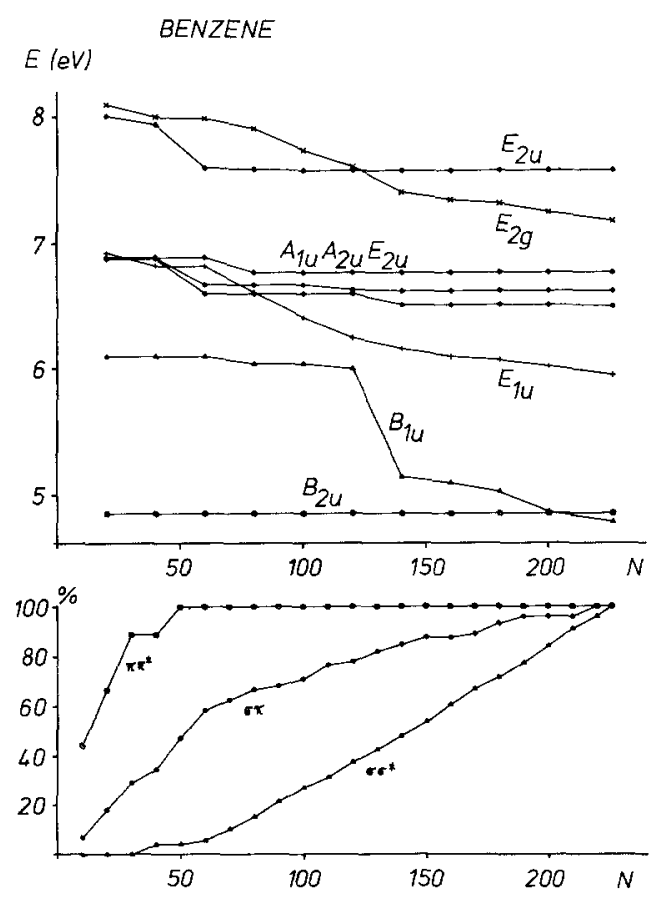

Fig. 5. Excitation energies of Benzene obtained from SCI/M calculations as function of the number of energy selected configurations. The lower part of the figure shows the percentage of $\pi \pi^{*}, \sigma \sigma^{*}$ and $\pi \leftrightarrow \sigma$ configurations, respectively, which are taken into account at a given number 
with the ZDO approximation. It should be mentioned that the result of a calculation where Pariser's $\gamma$-formula is used instead of $\mathrm{MN}$, is very similar for full SCI, though completely different at point 60 . The $\sigma \pi$-transitions change only little with further increase in the number of configurations, corresponding to the fact that at point 60 we already include about $60 \%$ of the possible $\sigma \pi$-configurations.

From this result, together with similar data for other systems, we have to suppose that the remarkable success of standard $\mathrm{CNDO} / \mathrm{S}$ in predicting excitation energies of low-lying DATs of unsaturated molecules is due to the fact that at about 60 SECs most of the $\pi \pi^{*}$ configurations but very little of the $\sigma \sigma^{*}$ ones are taken into account.

We now look to a similar plot for a SDCI calculation (Fig. 6). In this case Pariser's approximation is used for the $\gamma$-integrals because this calculations usually yield the best results when compared to experiment. As in the SCI case the low lying $\sigma \pi-$ transitions exhibit an excellent stability which is achieved very quickly. The $\pi \pi^{*}$ transitions also show a much better stability compared to the SCI results, in spite of the fact that benzene has been the worst of all our examples. Especially, the $1 B_{2 u}$ transition does not become reasonably stable below 200 configurations. In ZDO approximation this transition is only influenced by double excited configurations which interact either with the ground or with the excited state.

All other examples which we have investigated behave similar to hexatriene (Fig. 7). For more than about 150 energy selected configurations the calculated excitation energies vary only seldom by more than $0.3 \mathrm{eV}$. Above this number the point of

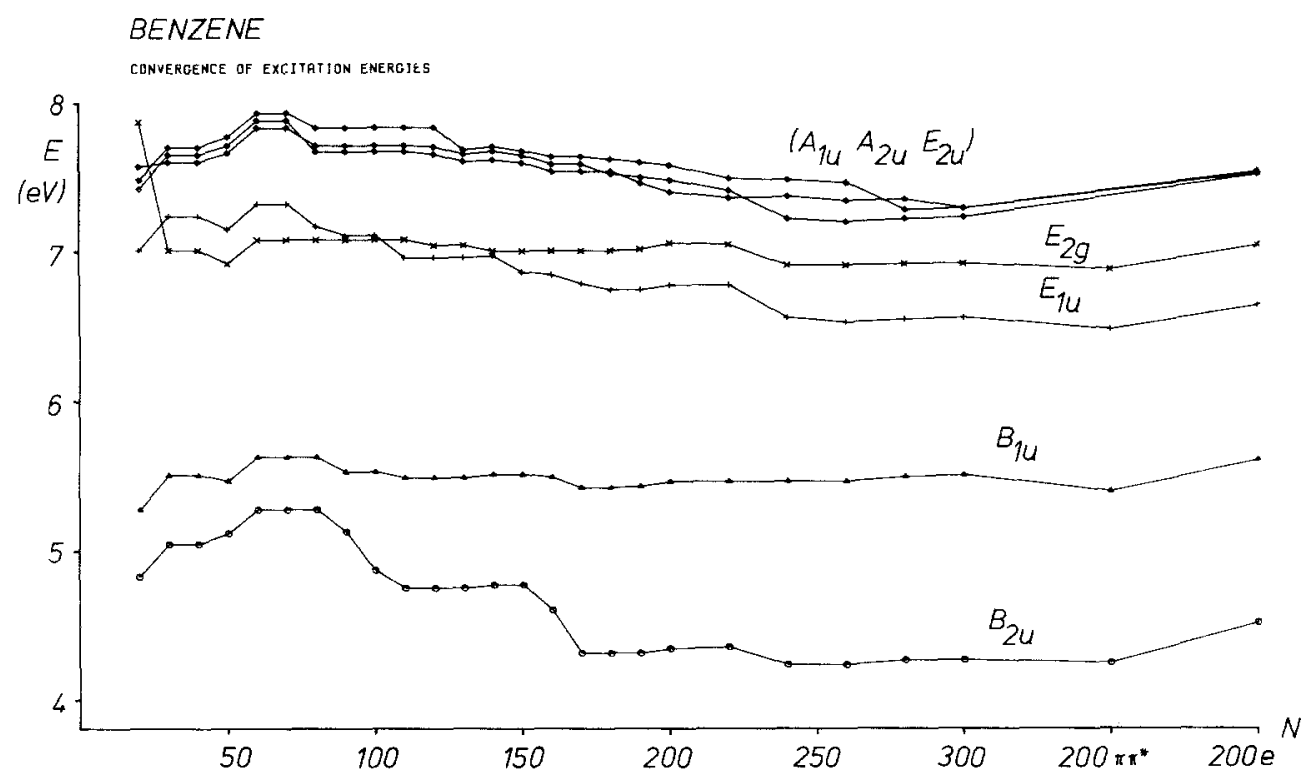

Fig. 6. Excitation energies of Benzene obtained from SDCI/P calculations as function of the number of energy selected configurations. Note change of scale in the abscissa after $N=300$; $200 e$ corresponds to a total number of 1600 and $200 \pi \pi^{*}$ to approximately 500 configurations 


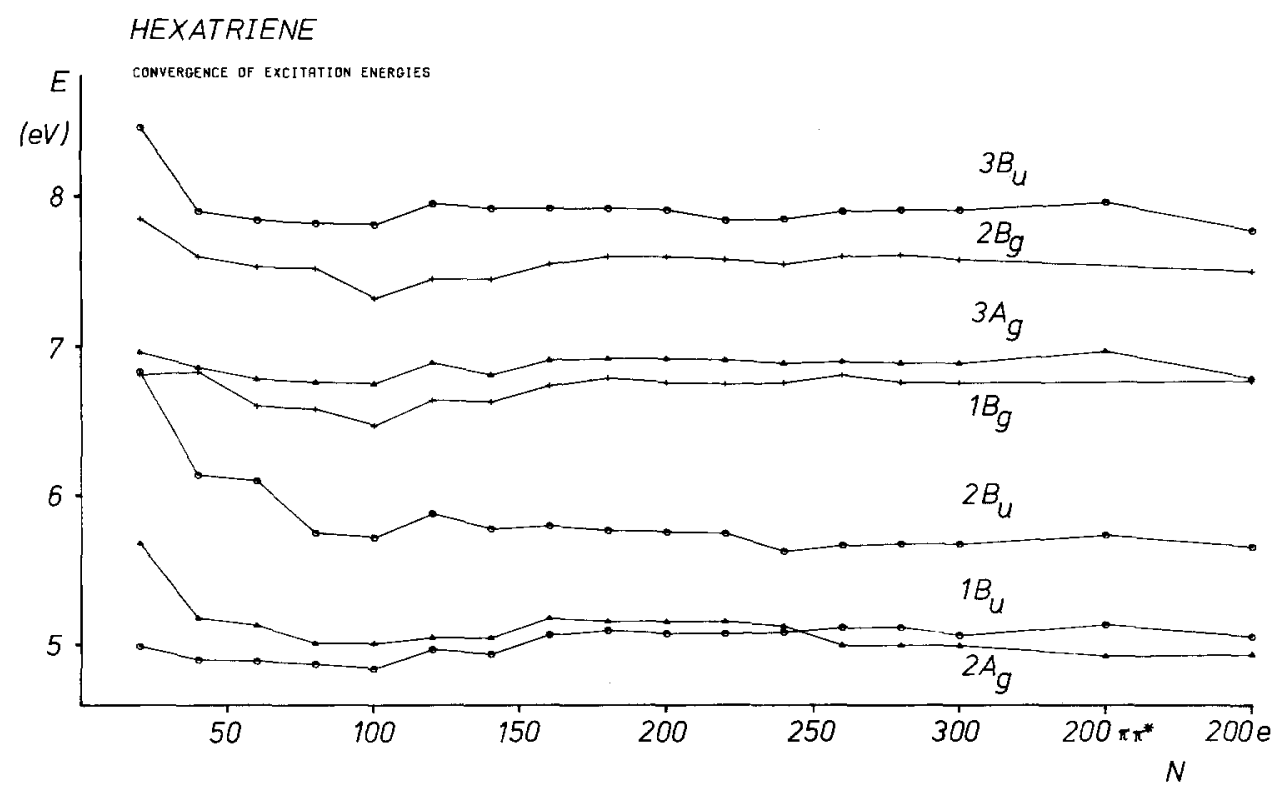

Fig. 7. Excitation energies of trans-1,3,5-hexatriene obtained from SDCI/P calculations as function of the number of energy selected configurations. $200 e$ corresponds to a total number of 800 in this case

truncation has no serious effect on the final result. Such a stability can only be understood if configurations of higher energy interact in a similar way with the low-lying excited states like they do with the ground state.

From our results we have to conclude that $\mathrm{CNDO} / \mathrm{S}$ calculations which include single and double excited configurations lead to reasonable stable results if at least about 200 energy selected configurations are taken into account. For molecules of high symmetry it seems to be necessary to use a somewhat larger CI basis. This is especially true if one of the low-lying excited states does not interact with higher SECs as in the case of the $1 B_{2 u}$-transition in benzene.

\section{Correlation Effects}

As mentioned in Sect. 3 it is by no means obvious that the specific results which have been obtained from PPP theory by application of $\gamma$-functions of different steepness [55] can be transferred to CNDO- or INDO-type methods. For a very short range effective Coulomb repulsion (ECR) a resemblance between the states obtained from these methods and the ionic and covalent states of VB theory must still exist. However, if the ECR is of intermediate range the lack of pairing could very well destroy this resemblance. It is therefore questionable if NDO-type methods also yield groups of states which exhibit such a different sensitivity toward correlation effects, as it has been found for the PPP results.

To gain better insight in this problem we have studied a number of examples where we performed calculations with the four different $\gamma$-functions mentioned in Sect. 2. The steepness of these functions increases in the order $\mathrm{OK} \approx \mathrm{P}<\mathrm{MN}<\mathrm{S}$. 
To avoid any mixing of different effects all other parameters, especially the " $\pi$ parameter" $\kappa$ were kept as in standard CNDO/S. Probably these parameters, too, have to be slightly adjusted if HEC are taken into account. It is, however, not the aim of the present investigation to obtain optimal coincidence with experimental values. It is our intention to study the general applicability of an extended allvalence-electron method which includes DEC to the simultaneous calculation of dipole allowed and dipole forbidden transitions. If the general applicability is shown, further improvements can be made by better adjustment of the remaining parameters.

As the general result is similar for all our examples it is sufficient to discuss hexatriene (Fig. 8) and benzene (Fig. 9) once again. The right side of each column shows the energies of the low-lying singlet states obtained from an SDCI calculation with 200 energy-selected configurations in each irreducible representation $(200 \mathrm{e})$. On the left side we give the result for the corresponding SCI calculation where all SECs are taken into account which appear in the SDCI treatment. In spite of the deviations caused by the different parametrization schemes, one clearly discerns two types of states. For the first type, the influence of DECs is only moderate and does not increase very much if we go from a less steep $\gamma$-function (like $O K$ ) to a very steep one (like S). For the second type the influence of DECs is stronger and increases rapidly with increasing steepness of the $\gamma$-function. Besides the ground state the low-lying excited states $2 A_{g}, 2 B_{u}$ and $4 A_{g}$ of hexatriene and $1 B_{2 u}$ and $1 E_{2 g}$ of benzene belong to the second type. From an inspection of the $\mathrm{CI}$ coefficients

\section{HEXATRIENE CNDO/S}

$$
\text { OHNO PARISER MATAGA SLATER }
$$
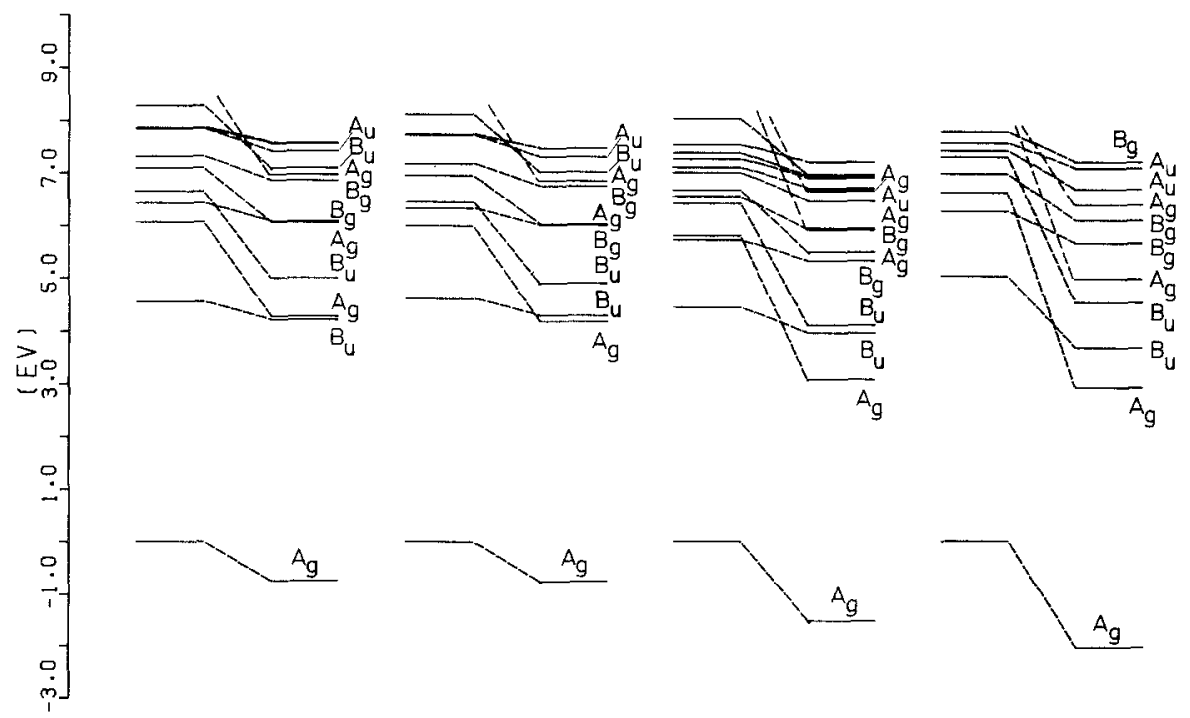

Fig. 8. Trans-1,3,5-hexatriene. Comparison of SCI (left) and SDCI (right) results for different $\gamma$-approximations 


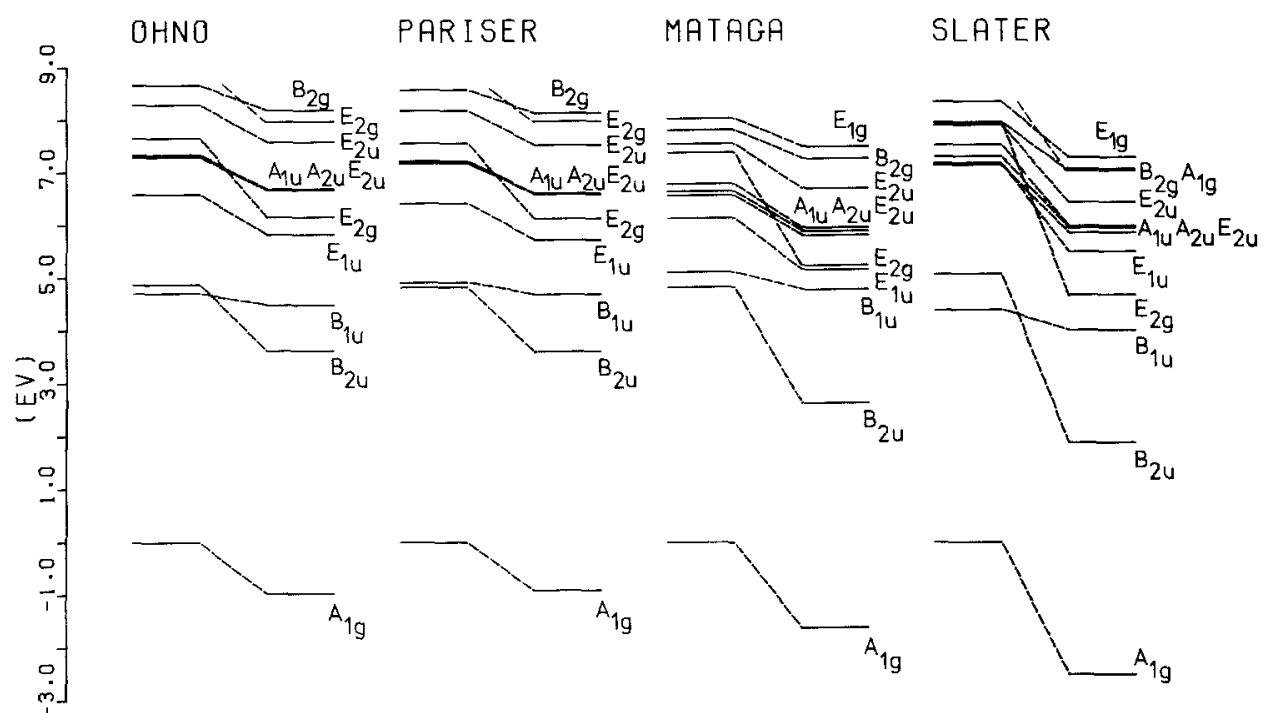

Fig. 9. Benzene. Comparison of SCI (left) and SDCI (right) results for different $\gamma$-approximations

it becomes obvious that all these states are related to minus-states of PPP theory. We find, that in CNDO-type methods (and probably in other NDO theories) the low-lying excited states still form two subsets which exhibit a different sensitivity towards correlation effects. At least these states seem to remember their parentage from ionic or covalent states up to an ECR of very moderate range. This resemblance is not connected with perfect pairing but a relation to the plus and minus states, which appear in theories with this type of pairing, is still apparent.

The practical conclusion drawn from this result is the following:

If the low-lying excited states form two subsets which depend in a different way on the actual range of the ECR the choice of an appropriate $\gamma$-approximation is of the same importance in CNDO/S-type methods as it is in PPP theories as soon as we include higher excited configurations. The proper choice of this approximation is of special importance if we want to describe transitions from the ground state to excited states which belong to different subsets at a similar level of accuracy.

\section{Comparison with Experiment}

To obtain a more detailed information on the type of $\gamma$-function which has to be used if DECs are included in CNDO/S-type calculations, we have investigated a number of examples. As it is our main goal to describe dipole allowed and dipole forbidden transitions at a comparable level of accuracy, we have to be aware of the following restrictions:

1. At least some experimental knowledge on low-lying DFTs should be available. As mentioned in the introduction, experiments in this field are still scarce; therefore, the number of possible examples is strongly reduced. 
2. DATs and DFTs undergo different solvent shifts. For a DAT with $f \simeq 1$ the shift between gas phase and solution is in the order of about $0.3 \mathrm{eV}$ whereas for a DFT it is usually less than $0.1 \mathrm{eV}$. If theoretical and experimental results are compared for both types of transition these solvent shifts must be considered. As far as possible we have used data from gas phase measurements throughout this paper to keep away from this problem.

For transitions for which an assignment is fairly certain the experimental energies are given in Tables 2-10 together with some of our theoretical results. The multiplicity index has been dropped due to the fact that we only discuss singlet excitations.

$\gamma$-integrals, which are calculated directly from Slater orbitals, yield even more pronounced correlation effects in SDCI calculations than the MN approximation. We therefore, skipped these data from presentation. We also do not show the SDCI results calculated with the OK relation because these results are always very similar to those obtained by application of Pariser's approximation (compare Figs. 8 and 9). As mentioned in Sect. 4 the excitation energies are shown for three different calculations in each case, where the same $\gamma$-approximation is used (P) but an increasing number of configurations. For the SDCI/P/200 $\pi \pi^{*}$ calculations we also show calculated oscillator strengths $(f)$ and two-photon cross sections $\left(\delta_{\uparrow \uparrow}\right)$. The $f$-and $\delta$-values for $\sigma \pi$-transitions given in brackets are from the SDCI/P/200t calculations. A detailed discussion of two-photon transition properties is given in part II of this series [4].

For all SDCI calculations the ground state depression is shown together with the excitation energies which refer to the actual ground state in each case. For comparison, we also present results of standard CNDO/S calculations with $60 \mathrm{SEC}$ and $\mathrm{MN}$ approximation for the $\gamma$-integrals (SCI/M/60).

\subsection{Trans-1,3-Butadiene}

Butadiene is one of the simplest unsaturated molecules. In spite of this fact, the assignment of the experimental spectrum is still under discussion. A recent reinvestigation of this spectrum by McDiarmid [61] leads to the following results: For the first diffuse band, which has its Franck-Condon Maximum (FCM) at $5.92 \mathrm{eV}$, the assignment as an NV transition has been confirmed. All the other features which can be observed below $8.3 \mathrm{eV}$ have been assigned to Rydberg transitions. A diffuse optical transition which had been proposed to exist around $1700 \AA(7.3 \mathrm{eV})$ from electron impact measurements [62] appears questionable due to this new investigation.

Butadiene, similar to hexatriene, does not exhibit any detectable emission. Twophoton absorption (TPA) measurements are therefore not possible with the usual detection technique and no information is available on two-photon cross sections (TPC) up to now. 

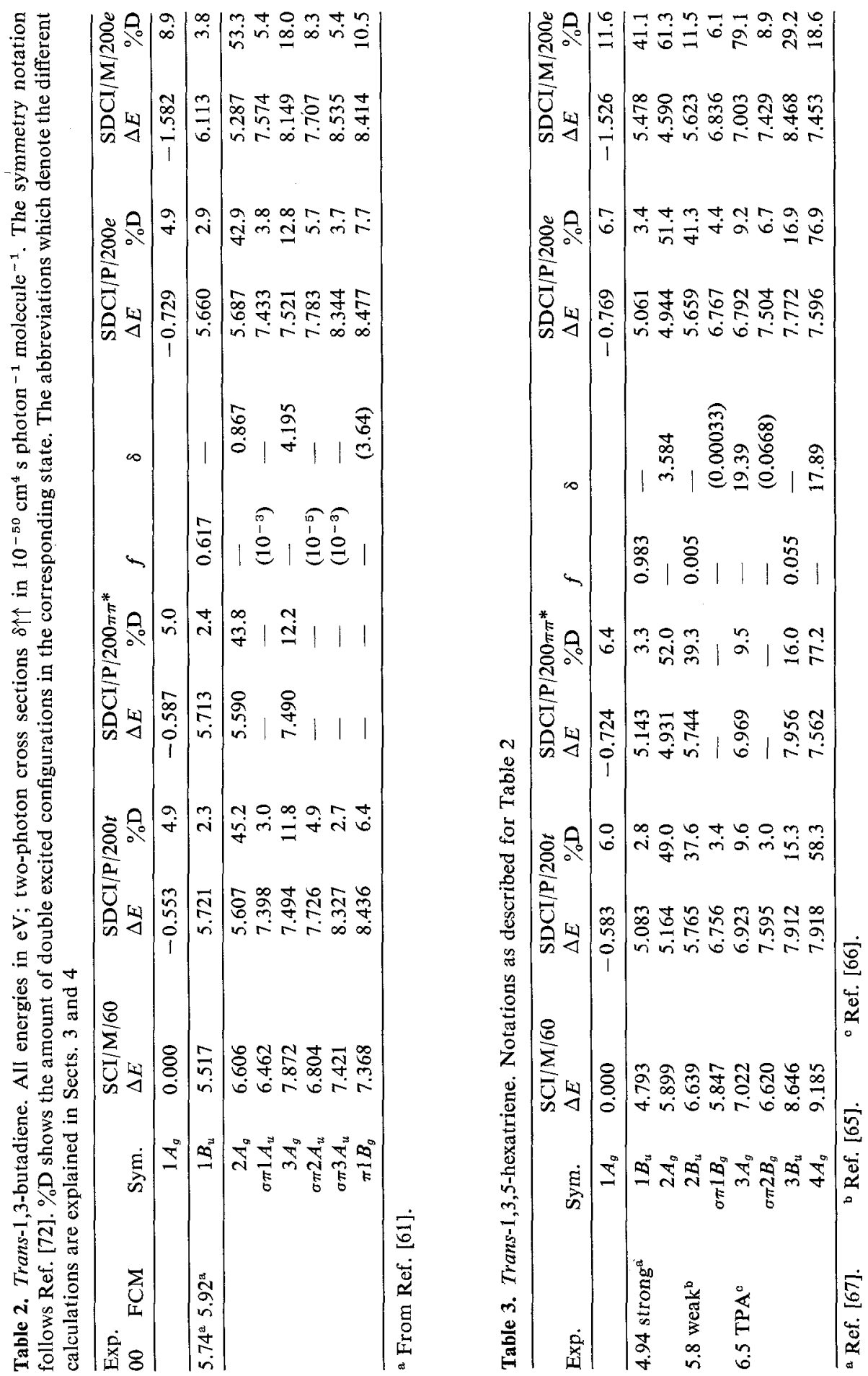
Ab initio calculations with extensive CI [63] lead to the result that all low-lying states, except $2 A_{g}$, have Rydberg character. The first singlet NV transition of symmetry $B_{u}$ is calculated to lie at $7.27 \mathrm{eV}$. To compare with the experimental energy it is assumed that the transition $1 B_{u} \leftarrow 1 A_{g}$ is extremely non-vertical. However, the overall intensity of the band between 5.5 and $6.5 \mathrm{eV}$ as well as its vibrational structure do not agree with such an assumption. Recent investigations have also shown that in the case of ethylene further extension of the basis set leads to considerable loss of Rydberg character in some of the low-lying excited states [64].

Our calculations (Table 2) yield three $\pi \pi^{*}$ - and four $\sigma \pi$-transitions in the energy range below $8.5 \mathrm{eV}$. The results are very stable with respect to further increase of the number of configurations. By inclusion of DECs all $\sigma \pi$-transitions are shifted by about $1 \mathrm{eV}$ to higher energies but they show very little dependence on the actual form of the $\gamma$-function. This is a result which we find for all the other examples, too. Therefore, we do not discuss it explicitly in the other cases. For the $\pi \pi^{*}$ transitions the influence of different $\gamma$-approximations is very pronounced. Due to the increasing ground state depression with increasing steepness of the $\gamma$-function transitions to the states $1 B_{u}$ and $3 A_{g}$ are shifted to higher energies. On the contrary, the $2 A_{g} \leftarrow 1 A_{g}$ transition is strongly lowered in energy by inclusion of DECs and this shift increases in going from $\mathrm{P}$ to $\mathrm{MN}$ approximation. The covalent character of the $2 \mathrm{~A}_{g}$ state becomes obvious from the high contribution of DECs $(\approx 50 \%)$ in the SDCI calculations.

As a consequence of the different shifts we obtain different qualitative results: SDCI calculations using Pariser's approximation predict the famous $2 A_{g} \leftarrow 1 A_{g}$ transition to lie very close to the allowed $1 B_{u}<-1 A_{g}$, whereas an application of the $\mathrm{MN}$ function yields this transition far in front $(0.8 \mathrm{eV})$ of $1 B_{u} \leftarrow 1 A_{g}$. Due to the lack of experimental information we are not able to discern between the two models in this case. The only certain experimental feature, the transition $1 B_{u} \leftarrow-1 A_{g}$ is predicted reasonably well by both models.

There is another point to be mentioned. The amount of DECs which contribute to $3 \mathrm{~A}_{g}$ together with the relative shift of the corresponding transition allow us to classify $3 A_{g}$ as "ionic" which always corresponds to "plus" in PPP theory. A two-photon transition from the ground state to a plus state is forbidden by plus/ minus selection rules. In our treatment, however, the lack of pairing destroys the plus character of $3 A_{g}$ to such an extent that $3 A_{g} \leftarrow 1 A_{g}$ becomes the most intense two-photon transition in the low energy region.

\subsection{1,3,5-Hexatriene}

Hexatriene is a much more favourable example as far as the experimental situation is concerned. Not only the recent and detailed study of the absorption spectra of trans- and cis-1,3,5-hexatriene by Gavin and Rice [65] is available, but also a TPA spectrum measured by Twarowski and Kliger [66] who used the so-called "twophoton thermal blooming technique", a technique applicable to non-luminescent molecules. 
Table 4. Cis-1,3,5-hexatriene. Notations as described for Table 2

\begin{tabular}{|c|c|c|c|c|c|c|c|c|}
\hline \multirow[b]{2}{*}{ Exp. } & \multirow[b]{2}{*}{ Sym. } & \multirow{2}{*}{$\frac{\mathrm{SCI} / \mathrm{M} / 60}{\Delta E}$} & \multicolumn{2}{|c|}{$\mathrm{SDCI} / \mathrm{P} / 200 t$} & \multicolumn{3}{|c|}{$\mathrm{SDCI} / \mathrm{P} / 200 \pi \pi^{*}$} & \multirow[b]{2}{*}{$\delta$} \\
\hline & & & $\Delta E$ & $\% \mathrm{D}$ & $\Delta E$ & $\% \mathrm{D}$ & $f$ & \\
\hline & $1 A_{1}$ & 0.000 & -0.587 & 6.1 & -0.724 & 6.5 & - & $\underline{-}$ \\
\hline \multirow[t]{2}{*}{4.92 strong $^{\mathrm{a}}$} & $1 B_{2}$ & 4.722 & 5.002 & 3.2 & 5.081 & 3.6 & 0.879 & 0.007 \\
\hline & $2 A_{1}$ & 5.734 & 5.032 & 47.5 & 4.807 & 50.5 & $7 \times 10^{-4}$ & 1.094 \\
\hline \multirow[t]{7}{*}{5.8 weak $^{\text {b }}$} & $2 B_{2}$ & 6.683 & 5.746 & 38.5 & 5.728 & 40.9 & $6 \times 10^{-4}$ & 1.829 \\
\hline & $3 A_{1}$ & 6.955 & 6.952 & 10.4 & 6.961 & 11.2 & 0.052 & 22.80 \\
\hline & $\sigma \pi 1 B_{1}$ & 6.398 & 7.190 & 2.7 & - & - & $(0.00018)$ & $(0.0022)$ \\
\hline & $\sigma \pi 1 A_{2}$ & 6.299 & 7.203 & 3.0 & - & 一 & $(0.0)$ & $(0.0139)$ \\
\hline & $\sigma \pi 2 A_{2}$ & 6,695 & 7.649 & 3.2 & - & - & $(0.0)$ & $(0.0551)$ \\
\hline & $3 B_{2}$ & 8.503 & 7.820 & 13.4 & 7.910 & 14.6 & 0.117 & 0.121 \\
\hline & $4 A_{1}$ & 9.245 & 7.881 & 56.4 & 7.573 & 75.2 & 0.0044 & 11.26 \\
\hline
\end{tabular}

${ }^{\mathrm{a}}$ Ref. [67]. ${ }^{\mathrm{b}}$ Ref. [65].

The combined experimental information of these investigations reads as follows (compare Tables 3 and 4): In each case the spectrum starts with an intense band. The 00-transition of this band shows very similar energies in trans- and cis-hexatriene. A weak band is proposed to start at about $5.8 \mathrm{eV}$ in the spectra of both isomers. A third medium intense band has its origin at $6.54 \mathrm{eV}$ in trans- and $6.14 \mathrm{eV}$ in cis-hexatriene. Another band of medium intensity is proposed to start at about $7.3 \mathrm{eV}$ in the trans and at $7.03 \mathrm{eV}$ in the cis isomer.

The TPA spectrum shows a first increase between 5.0 and $5.9 \mathrm{eV}\left(\mathrm{TPC} \approx 2 \cdot 10^{-50}\right.$ $\mathrm{cm}^{4} \mathrm{~s}$ photon ${ }^{-1}$ molecule ${ }^{-1}$ at $5.21 \mathrm{eV}$ ) and a further rapid increase towards the experimental limit of the spectrum at $6.5 \mathrm{eV}\left(\mathrm{TPC} \geqslant 30 \cdot 10^{-50} \mathrm{~cm}^{4} \mathrm{~s}\right.$ photon ${ }^{-1}$ molecule ${ }^{-1}$ ). The second increase coincides well with the band starting at $6.54 \mathrm{eV}$ in trans-hexatriene. Unfortunately, Twarowski and Kliger do not know very precisely the amount of $c i s$-isomer in their sample.

As far as the influence of the $\gamma$-function is concerned, our theoretical results are similar to those obtained for butadiene (Table 2). The steeper MN approximation predicts not only the $2 A_{g}$ level to be separated by about $1 \mathrm{eV}$ from the lowest $B_{u}$ level but also an interchange of the character of the first two $B_{u}$ states. A covalent $B_{u}$ state related to a $B_{u}^{(-)}$is now lying in front of the first ionic $B_{u}$ state. A similar interchange of ionic and covalent character occurs between $3 A_{g}$ and $4 A_{g}$ if we go from the $\mathrm{P}$ to the $\mathrm{MN}$ approximation. For hexatriene the steeper $\mathrm{MN}$ function leads to unreasonable results if compared with experiment. We therefore restrict our further discussion to the $P$ results.

A comparison of the calculated energies of the trans (Table 3) and cis (Table 4) isomer shows that the first $\sigma \pi$-transitions are somewhat higher in the cis isomer but that the low-lying $\pi \pi^{*}$-transitions have practically the same energies in both forms. At least for the first two bands this is in accordance with the experimental observation. If the second band is assigned to $2 B_{u} \leftarrow 1 A_{g}$ in trans- and to $2 \mathrm{~B}_{2} \leftarrow 1 A_{1}$ in $c i s$-hexatriene the calculated energies and intensities compare extremely well with the experimental data (intensity ratio trans/cis: calculated 1.12, experimental 
$1.1[67])$. The same assignment has already been proposed by Karplus, Gavin and Rice [68].

Similar to butadiene our calculations predict the transitions $2 A_{g} \leftarrow 1 A_{g}$ (or $2 A_{1} \leftarrow$ $1 A_{1}$ ) and $1 B_{u} \leftarrow 1 A_{g}$ (or $1 B_{2} \leftarrow 1 A_{1}$ ) to be close in energy. Only steep $\gamma$-functions which yield unreasonable results for the first two $B_{u}$ states lead to a well separated $2 A_{g}$-transition as it is found in PPP calculations [68] also for $\gamma$-functions like OK. From this it is not surprising that no indication of a forbidden $A_{g} \leftarrow A_{g}$ transition has been found on the low energy tail of the first band in trans-hexatriene [67]. It is much more likely that the increase in two-photon absorption at about $5.0 \mathrm{eV}$ has to be attributed to the $2 A_{g} \leftarrow 1 A_{g}$ transition and that this transition is superimposed by the allowed $1 B_{u} \leftarrow 1 A_{g}$ transition [4].

The second increase in TPA fits very well with the high $\delta$-value which we obtain for $3 A_{g} \leftarrow 1 A_{g}$. Twarowski and Kliger [66] who calculated TPC in PPP approximation.including DEC were not able to give any theoretical explanation for a strongly allowed two-photon transition below $8.5 \mathrm{eV}$. As in butadiene $3 A_{g} \leftarrow 1 A_{g}$ is two photon forbidden in a PPP-type calculation due to plus/minus selection rules.

Some unsolved problems in the assignment of those bands which appear at higher energies in the spectra of cis- and trans-hexatriene $[65,68]$ prohibit a further discussion of this energy range in the context of this paper.

\subsection{Trans-1,3,5,7-Octatetraene}

The theoretical results (Table 5) are basically the same as for the other polyenes. One half of the low-lying states is strongly influenced by DECs $(30-60 \% \mathrm{D})$. This influence increases with increasing steepness of the $\gamma$-function leading again to unreasonable results for a $\gamma$-approximation like MN. Also in this case the first covalent $B_{u}$ state is found to have a lower energy than the first ionic one in SDCI/M calculations.

The SDCI/P calculations yield an energy of about $4.6 \mathrm{eV}$ which compares well with the experimental value of $4.4 \mathrm{eV}$ [69]. The $2 A_{g} \leftarrow 1 A_{g}$ transition is predicted to lie at only somewhat lower energies, a result which does not agree with the recent experimental investigation of Gavin, Weisman, McVey and Rice [69]. These authors claim to have identified the $2 A_{g}$ transition from absorption and fluorescence measurements made in solution. The 00 transition, extrapolated to gas phase is proposed to lie at $3.594 \mathrm{eV}$. This band could, however, not be found in the gas phase absorption spectrum and-what is more surprising-the gase phase fluorescence seems to come from the $1 B_{u}$-state but with a radiative life time which does not fit to an allowed $1 B_{u} \leftarrow 1 A_{g}$ transition. A gap of $2500 \mathrm{~cm}^{-1}$ between $1 A_{g}$ and $1 B_{u}$ has been observed by Andrews and Hudson [70] for deca-2,4,6,8-tetraene in $n$-alkane matrix at $4.2 \mathrm{~K}$. Taking into account solvent shifts this should lead to a gap of about $0.5-0.6 \mathrm{eV}$ in gas phase, but here we do not know the specific influence of the methyl-groups to the individual states.

To clarify the real distance between $2 A_{g}$ and $1 B_{u}$ two-photon absorption measurements are highly desirable. The calculated $\delta$-value which is twice as large as for 







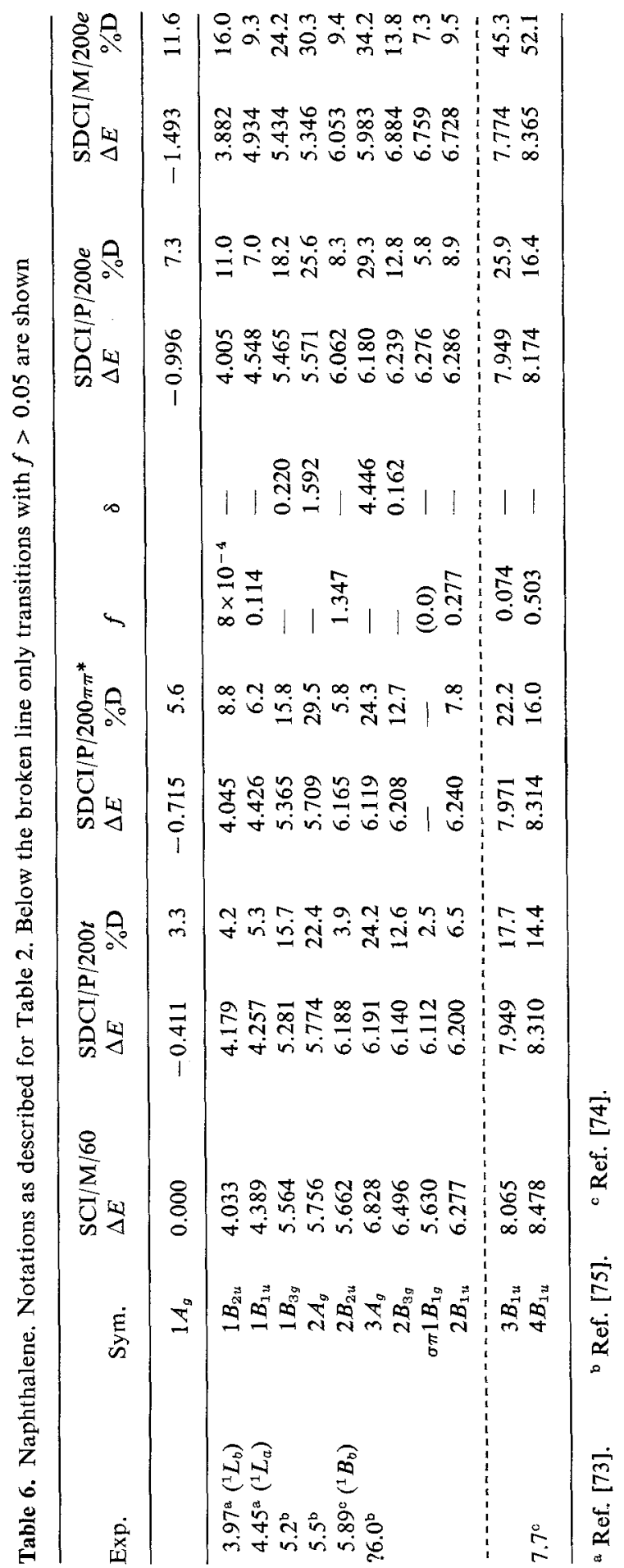




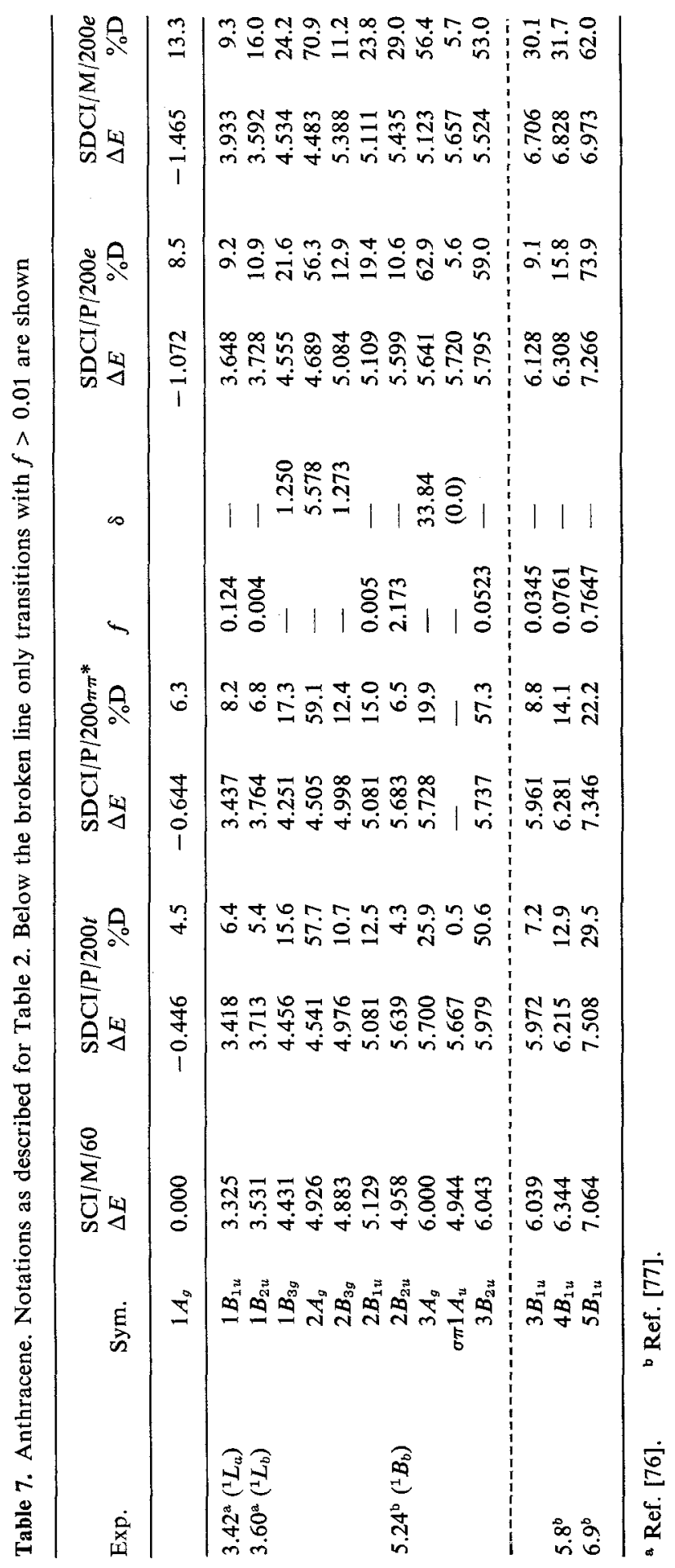


the same transition in hexatriene should be stimulating for such a task. A second covalent $A_{g}$ state $\left(3 A_{g}\right.$ ) is calculated in the low energy region for trans-octatetraene but the largest two-photon cross section is still obtained for $4 A_{g} \leftarrow 1 A_{g}$, a transition which leads into the first ionic state of this symmetry.

Two further bands have been identified at higher energies by Gavin et al. [69]: A weak one, starting at about $5.3 \mathrm{eV}$ and a medium intense one with a proposed origin at $5.694 \mathrm{eV}$. None of these bands seems to be of Rydberg origin. The first of the two bands fits well to an assignment $2 B_{u} \leftarrow 1 A_{g}$. The second, however, is difficult to assign because we do not find any transition of medium intensity below $7.3 \mathrm{eV}$. This is similar to the problem still apparent in trans-1,3,5-hexatriene.

The basic results for the next few examples-condensed benzoide hydrocarbonsdiffer considerably from those obtained for the polyenes. The influence of HEC is by far less pronounced for this type of molecules (Tables 6-8). As suggested earlier only some of the states which give rise to DFT from the ground state are afflicted more seriously by inclusion of DECs but not as strong as in the case of the polyenes.

The resemblance between covalent and minus states is not so obvious. Even the ${ }^{1} L_{b}$-transitions (the well established nomenclature of Platt [71] is used together with the symmetry notation [72] for the benzoide hydrocarbons), transitions which have to be classified as "minus" in PPP theory, do not gain larger contributions of DECs. Only for steep $\gamma$-functions the covalent character of some of the low-lying states becomes more pronounced as we can see from the SDCI/M results. These results, however, lead to a less favourable agreement with experiment. We therefore conclude that also for the benzoid hydrocarbons steep $\gamma$ functions like MN overestimate correlation effects in SDCI calculations.

Similar to the polyenes the lowest $\sigma \pi$-transitions are raised by about $0.7 \mathrm{eV}$ if DECs are included.

\subsection{Naphthalene}

For the states which give rise to the well-known optical transitions ${ }^{1} L_{b},{ }^{1} L_{a}$ and ${ }^{1} B_{b}$ the results of our SDCI/P calculations are in similar agreement with experiment

Table 8. Phenanthrene. Notations as described for Table 2

\begin{tabular}{llllrrrrl}
\hline & & \multicolumn{3}{l}{$\mathrm{SCI} / \mathrm{M} / 60$} & \multicolumn{2}{l}{$\mathrm{SDCI} / \mathrm{P} / 200 t$} & \multicolumn{3}{l}{$\mathrm{SDCI} / \mathrm{P} / 200 \pi \pi^{*}$} \\
Exp. & $\mathrm{Sym}$. & $\Delta E$ & \multicolumn{1}{c}{$\Delta E$} & $\% \mathrm{D}$ & \multicolumn{1}{c}{$\Delta E$} & \multicolumn{1}{c}{$\% \mathrm{D}$} & \multicolumn{1}{l}{$f$} & \multicolumn{1}{l}{$\delta$} \\
\hline & $1 A_{1}$ & 0.000 & -0.340 & 3.0 & -0.629 & 5.1 & - & - \\
\hline $3.78^{\mathrm{a}}\left({ }^{1} L_{b}\right)$ & $2 A_{1}$ & 3.661 & 3.826 & 5.5 & 3.950 & 7.1 & - & 0.532 \\
$4.36^{\mathrm{a}}\left({ }^{1} L_{a}\right)$ & $1 B_{2}$ & 4.151 & 4.144 & 2.7 & 4.300 & 4.0 & 0.116 & - \\
& $2 B_{2}$ & 4.728 & 4.998 & 3.3 & 5.112 & 6.2 & 0.058 & 0.065 \\
$4.75^{\mathrm{b}}\left({ }^{1} B_{b}\right)$ & $3 A_{1}$ & 4.952 & 5.046 & 8.2 & 5.178 & 11.9 & 0.086 & 1.81 \\
$4.95^{\mathrm{b}}\left({ }^{1} B_{a}\right)$ & $3 B_{2}$ & 4.945 & 5.223 & 3.6 & 5.355 & 5.7 & 0.821 & $4 \times 10^{-4}$ \\
& $4 A_{1}$ & 5.208 & 5.369 & 14.8 & 5.473 & 18.6 & 0.173 & 4.77 \\
$5.63^{\mathrm{b}}$ & $5 A_{1}$ & 5.780 & 5.688 & 7.2 & 5.774 & 7.6 & 0.100 & 1.76 \\
$5.86^{\mathrm{b}}$ & $4 B_{2}$ & 5.695 & 5.834 & 6.9 & 5.870 & 13.0 & 0.223 & $8 \times 10^{-5}$ \\
& $5 B_{2}$ & 6.250 & 6.097 & 24.3 & 6.043 & 30.4 & 0.027 & 6.52 \\
\hline
\end{tabular}

${ }^{\mathrm{a}}$ Ref. [79]. ${ }^{\mathrm{b}}$ Ref. [25]. 
as the standard CNDO/S results (Table 6). At higher energies we find a transition with $f=0.28$ at about $6.2 \mathrm{eV}$ and another one with $f=0.50$ at about $8.3 \mathrm{eV}$ both leading to states of $B_{1 u}$-symmetry. All other transitions in this energy range have oscillator strength less than 0.1 . The $2 B_{1 u} \leftarrow 1 A_{g}$ transition is calculated to be close in energy to the strongly one-photon allowed transition $2 B_{2 u} \leftarrow 1 A_{g}$. Correspondingly this transition is not well resolved in the experimental spectrum but some indications are obtained for its existence [74]. The $4 B_{1 u}<-1 A_{g}$ transition corresponds very well with an intense band around $7.7 \mathrm{eV}$ in the gas phase spectrum. With increasing energy the calculated excitation energies are increasingly overestimated but the overall correlation shown in Fig. 10 also holds for these high energy excitations.

Two-photon spectroscopy [75] has revealed a weak two-photon allowed transition at $5.2 \mathrm{eV}$ and a stronger one at about $5.5 \mathrm{eV}$, both in agreement with our theoretical results. However, similar results are still obtained from standard CNDO/S calculations. The only transition which is considerably shifted by inclusion of DECs is $3 A_{g} \leftarrow 1 A_{g}$. The calculated TPC corresponds very well to an intense two-photon absorption around $6.0 \mathrm{eV}$, but the mechanism which is responsible for this absorption is not yet fully resolved (see part II for further discussion).

\subsection{Anthracene}

Similar to naphthalene the energies of the dipole allowed transition are not changed very much if we go from standard CNDO/S to SDCI/P (Table 7). The

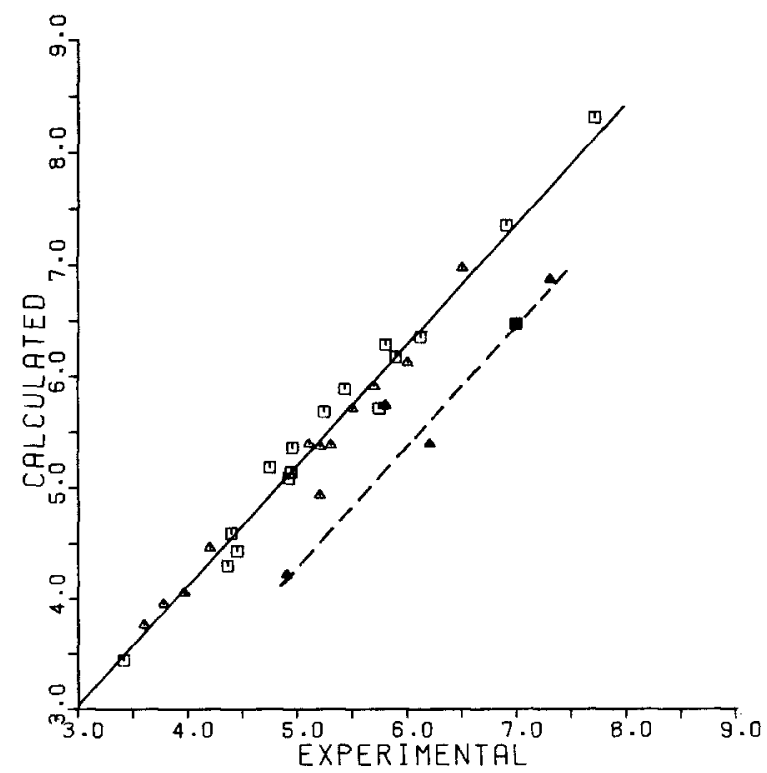

Fig. 10. Comparison between experimental and calculated excitation energies. The theoretical data are from the $\mathrm{SDCI} / 200 \pi \pi^{*}$ calculations. $\square$ dipole allowed transitions. $\triangle$ dipole forbidden transitions. The data of Benzene (filled symbols) do not fit into the general correlation (see text). If these data are omitted the correlation is given by $E_{\mathrm{cal}}=1.087 E_{\mathrm{exp}}-0.252$ with a correlation coefficient $R=0.985$ 
only major deviation is found for $2 B_{2 u} \leftarrow 1 A_{g}$ the energy of which is somewhat underestimated by CNDO/S and somewhat overestimated by SDCI/P. The latter result, however, fits perfectly to the overall correlation shown in Fig. 10. In the high energy region two weak to medium intense transitions leading into states of $\mathrm{B}_{1 u}$ symmetry are calculated at about 6.0 and $6.3 \mathrm{eV}$. This corresponds to a structure at about $5.8 \mathrm{eV}$ in the gas phase absorption spectrum which has been assigned to a $B_{1 u} \leftarrow 1 A_{g}$ transition by comparison with solution and solid state spectra [77]. The next transition for which a higher oscillator strength is calculated is $5 B_{1 u} \leftarrow 1 A_{g}$ at $7.3 \mathrm{eV}$ fitting perfectly to an intense transition of $B_{1 u}$-symmetry observed in gas phase absorption around $6.9 \mathrm{eV}$.

Standard CNDO/S predicts three states of $g$-symmetry to lie in the region between ${ }^{1} \mathrm{~L}_{b}$ and ${ }^{1} B_{b}$. One of the corresponding states $\left(2 A_{g}\right)$ is obviously of covalent nature $(50 \% \mathrm{D})$ and therefore, considerably stabilized by inclusion of HECs. Strong twophoton absorption is indeed observed between 4 and $5 \mathrm{eV}$ [78] but the experimental information is not sufficient to decide whether $2 A_{g} \leftarrow 1 A_{g}$ is close to the strongly one-photon allowed ${ }^{1} B_{u}$-transition as predicted by the SCI-results or halfways in between ${ }^{1} L_{a}$ and ${ }^{1} B_{b}$ as proposed by our SDCI calculations [4].

\subsection{Phenanthrene}

From all our examples phenanthrene is the one which shows the smallest influence of HECs. Correspondingly the SDCI results differ only in minor details from those of standard CNDO/S calculations. A state of $A_{1}$ symmetry $\left(3 \mathrm{~A}_{1}\right)$ is found to lie somewhat below $3 B_{2}$ as proposed earlier from conventional polarization spectroscopy [25] and from two-photon absorption [3c]. The medium intense transitions $5 A_{1} \leftarrow 1 A_{1}$ and $4 B_{2} \leftarrow 1 A_{1}$ correspond to structures around 5.6 and $5.8 \mathrm{eV}$ in the experimental spectrum. As these values are from solution spectra a solvent shift of about $0.2 \mathrm{eV}$ must be added to compare with the other experimental data. Polarization measurements indicate [25] that the transition at about $5.6 \mathrm{eV}$ is indeed of $A_{1}$ symmetry.

\subsection{Benzene}

Benzene is the example of the classical investigation by Koutecký and coworkers [46] where the different sensitivity of different groups of excited states towards correlation effects and the importance of the steepness of the $\gamma$-function has been fully recognized for the first time. Although the influence of HECs is not as pronounced for benzene as for polyenes the resemblance between minus and covalent states is more obvious than in the case of condensed benzoide hydrocarbons. With increasing steepness of the $\gamma$-function inclusion of DECs leads to a widening of the gap between ${ }^{1} L_{b}$ and ${ }^{1} L_{a}$ and a closure of the gap between ${ }^{1} B_{a, b}$ and $1 E_{2 g} \leftarrow 1 A_{1 g}$. This is due to the increasing stabilisation of the "minus" states $1 A_{1 g}, 1 B_{2 u}$ and $1 E_{2 g}$ (compare Fig. 9). The $1 E_{2 g} \leftarrow 1 A_{1 g}$ transition has been proposed to lie at $7.3 \mathrm{eV}$ [82], an assignment which corresponds to an increase in two-photon absorption observed at about this energy [83]. It should be mentioned, 
however, that the interpretation of this absorption has been questioned recently [84].

Compared with the other results the calculated excitation energies are by far too low for benzene. If we include benzene into the correlation diagram of Fig. 10 where we compare the results of all our SDCI/P/200 $\pi \pi^{*}$ calculations to experimental excitation energies, the benzene values seem to lie at a separate line which has about the same slope as the main correlation line but a different onset. We also find a mean increase of $0.2 \mathrm{eV}$ for the calculated excitation energies without any major change in the internal distances if we go from the SDCI/P/200 $\pi \pi^{*}$ to the $\mathrm{SDCI} / \mathrm{P} / 200 \mathrm{e}$ calculation. This seems to indicate that 200 configurations in each irreducible representation is still not enough to describe the ground state properly. It will be interesting to study whether this is an effect generally observed for systems with degenerate one-electron levels.

\subsection{Stilbene}

Our last example (Table 10) can be looked at as the first member of the series of $\alpha, \omega$-diphenyl-polyenes, the systems which first gave evidence for the unsuitability of the simple one-electron excitation picture. The more astonishing it is, that in this case too, the influence of HECs on the calculated excitation energies is just little. The only state below $6 \mathrm{eV}$ which can obviously be labelled "covalent" due to the contribution of DECs is $4 A_{g}$.

At this point it should be mentioned that also for those systems where DECs yield only minor changes in excitation energies their inclusion is very important for the calculation of two-photon transition probabilities. This is discussed in detail in part II of this series.

The UV spectrum of stilbene exhibits three bands with Franck-Condon maxima at 4.2, 5.4 and $6.1 \mathrm{eV}$ in ethanol [85]. If these values are compared with calculated excitation energies a solvent shift of about $0.3 \mathrm{eV}$ has to be estimated for each transition. The three bands are attributed to transitions into the states $1 B_{u}, 3 B_{u}$ and $4 B_{u}$. In addition to these states the calculations predict another $B_{u}$ state $\left(2 B_{u}\right)$ and three $A_{g}$ states in the low energy region. A strong two-photon absorption has been observed [86] around $5.1 \mathrm{eV}$ in good agreement with the calculated data of $3 A_{g} \leftarrow 1 A_{g}$ [4]. There is also some indication for another $A_{g} \leftarrow A_{g}$ transition at lower energies in the TPA spectrum. Due to our calculations it should be very interesting to extend the TPA measurements towards higher energies where a transition with very large two-photon cross section is predicted around $5.8 \mathrm{eV}$.

\section{Conclusion}

The main purpose of this investigation was to study whether CNDO/S-type calculations can be used or not to predict excitation energies for dipole-allowed and dipole-forbidden transitions at a similar level of accuracy. We believe to have 


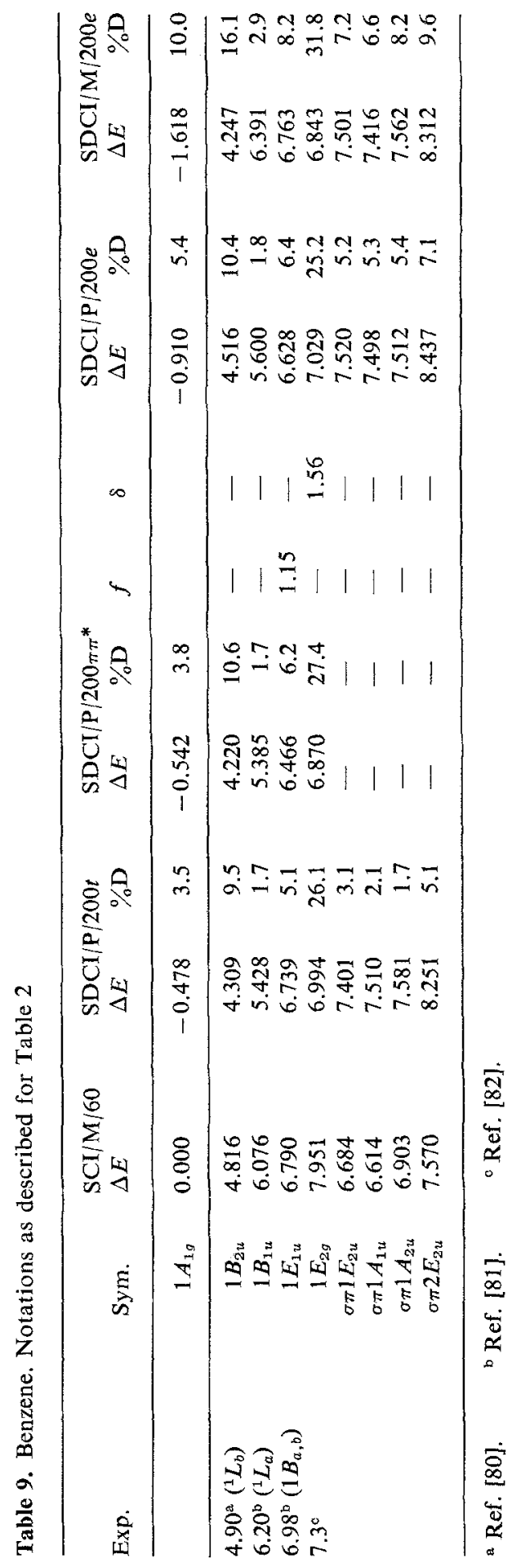




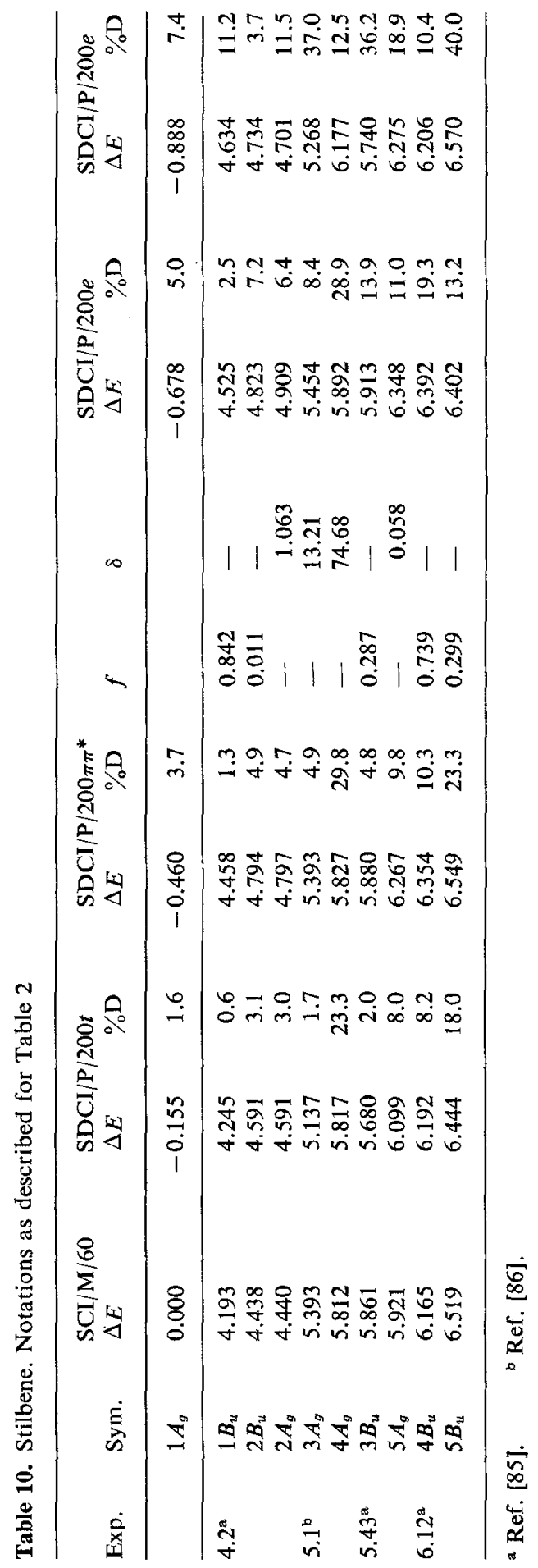


proved that this is indeed possible if DECs are included and if the importance of the $\gamma$-function is considered. From the SDCI/P/200 $\pi \pi^{*}$ calculations, an overall correlation (Fig. 10) is obtained which is very promising. Further improvement should be possible by careful readjustment of those parameters which we have adopted unchanged from standard $\mathrm{CNDO} / \mathrm{S}$. The main results of our investigation may be summarized as follows:

a) Also in CNDO-type methods, where the pairing theorem is no longer valid, we are usually able to distinguish two groups of excited states, one of which is more sensitive towards correlation effects than the other. The group with the higher sensitivity gives usually rise to transitions which are weak or forbidden in one-photon spectroscopy. If these states are of interest, DECs must be included.

b) The influence of DECs is by far less pronounced in condensed benzoid hydrocarbons than it is in linear chain systems (polyenes), but also for the benzoid hydrocarbons inclusion of DECs is necessary to obtain appropriate excitation energies for DFTs.

c) The resemblance between ionic and plus states, and covalent and minus states, respectively, is much more pronounced in polyenes than in benzoid hydrocarbons where the lack of pairing seems to destroy this resemblance very quickly.

d) Also in cases where DECs yield only little influence on excitation energies, their inclusion is very important for the calculation of two-photon transition properties as shown in part II of this series. Strong violations of the plus/minus selection rules are observed for these quantities.

e) Compared to the $\pi \pi$-manifold $\sigma \pi$-transitions are always shifted by at least $0.5 \mathrm{eV}$ to higher energy after inclusion of DECs.

f) CNDO/S-type calculations which include DECs exhibit a considerable stability of calculated excitation properties with increasing number of configurations if at least 200 energy selected configurations are taken into account.

g) As in PPP theory steep $\gamma$-functions like MN overestimate correlation effects if DECs are included. The choice of a proper $\gamma$-approximation is very important in this case.

Acknowledgement. Financial support for this project from the Deutsche Forschungsgemeinschaft is gratefully acknowledged. We also thank the Computer center of the University of Cologne for providing the necessary computing time. One of us (B. D.) also gratefully acknowledges a scholarship of Cusanuswerk.

\section{References}

1. Birks, J. B.: Organic molecular photophysics II, p. 99. London-New York-TorontoSydney: John Wiley

2. Trajmar, S., Rice, J. K., Kuppermann, A.: Advan. Chem. Phys. 18, 15 (1970)

3. McClain, W. M.: Accounts Chem. Res. 7, 129 (1974); Drucker, R. P., McClain, W. M.: J. Chem. Phys. 61, 2609 (1974); Drucker, R. P., McClain, W. M. : J. Chem. Phys. 61, 2616 (1974) 
4. Hohlneicher, G., Dick, B.: J. Chem. Phys., 70, 5427 (1979)

5. Pople, J. A.: Trans. Faraday Soc. 44, 1375 (1953)

6. Pariser, R.: J. Chem. Phys. 24, 250 (1956); Kohn, W.: Phys. Rev. A133, 171 (1964); McLachlan, A. D.: Mol. Phys. 2, 271 (1959); 4, 49 (1961)

7. Koutecký, J.: J. Chem. Phys. 44, 3702 (1966); 45, 3668 (1966)

8. Bulevskii, L. N.: Sov. Phys. JETP 24, 154 (1967); Visscher P. B., Falicov, L. M.: J. Chem. Phys. 52, 4217 (1970)

9. Bolton, J. R., Fraenkel, G. K.: J. Chem. Phys. 40, 3307 (1964)

10. Aalbersberg, W. I. J., Hoytink, G. J., Mackor, E. L., Weijland, W. P.: J. Chem. Soc. 3049 (1959)

11. Distler, D., Hohlneicher, G.: Chem. Phys. Letters 7, 345 (1970)

12. Ellis, R. L., Jaffé, H. H.: J. Mol. Spectry. 50, 474 (1974)

13. Parr, R. G., Pariser, R.: J. Chem. Phys. 21, 466 (1953)

14. Parr, R. G., Pariser, R.: J. Chem. Phys. 21, 767 (1953)

15. Clark, P. A., Ragle, J. L.: J. Chem. Phys. 46, 4235 (1967)

16. Del Bene, J., Jaffé, H. H.: J. Chem. Phys. 49, 1221 (1968); 48, 1807 (1968); 48, 4050 (1968); 50, 1126 (1969)

17. Lipari, N. O., Duke, C. B.: J. Chem. Phys. 63, 1748 (1975)

18. Zerner, M., Ridley, J.: Theoret. Chim. Acta (Berl.) 32, 111 (1973)

19. Krogh-Jespersen, K., Ratner, M.: Theoret. Chim. Acta (Berl.) 47, 283 (1978)

20. Pariser, R.: J. Chem. Phys. 21, 568 (1953)

21. Nishimoto, K., Mataga, N.: Z. Physik. Chem. 12, 335 (1957); 13, 140 (1957)

22. Ohno, K.: Theoret. Chim. Acta (Berl.) 2, 219 (1964)

23. Klopman, G.: J. Am. Chem. Soc. 86, 4550 (1964)

24. Lorquet, A. J.: Theoret. Chim. Acta (Berl.) 5, 192 (1966)

25. Dörr, F., Hohlneicher, G., Schneider, S.: Ber. Bunsenges. Physik. Chem. 70, 803 (1966)

26. Lo, D. H., Whitehead, M. A.: Can. J. Chem. 46, 2027 (1968)

27. Lo, D. H., Whitehead, M. A.: Can. J. Chem. 46, 2041 (1968)

28. Marschner, F., Goetz, H.: Tetrahedron 29, 3105 (1973)

29. Marschner, F., Goetz, H.: Tetrahedron 30, 3159 (1974)

30. Lipari, N. O., Duke, C. B.: J. Chem. Phys. 63, 1768 (1975)

31. Pohle, H.: Diss. Berlin 1976

32. Michl, J.: Intern. J. Quantum Chem. Symp. 10, 107 (1976)

33. Thulstrup, E. W., Downing, J. W., Michl, J.: Chem. Phys. 23, 307 (1977)

34. Loux, J. P.: Diss. Köln 1978

35. Roothaan, C. C. J.: J. Chem. Phys. 19, 1445 (1951)

36. Hohlneicher, G.: Habilitationsschrift, TU München 1967

37. Ellis, R. L., Jaffé, H. H., in: Modern theoretical chemistry, 8. Semiempirical methods of electronic structure calculations, A. Segal ed., p. 66ff. New York and London: Plenum Press 1977

38. Schneider, S.: Diplomarbeit, TU München 1965

39. Craig, D. P.: Proc. Roy. Soc. A200, 474 (1950)

40. Craig, D. P.: J. Chem. Phys. 17, 1358 (1949)

41. Parr, R. G., Craig, D. P., Ross, I. G.: J. Chem. Phys. 18, 1561 (1950)

42. Moser, C. M.: J. Chem. Soc. 3455 (1954)

43. Murrel, J. N., McEwen, K. L.: J. Chem. Phys. 25, 1143 (1956)

44. Bloor, J. E., Lee, J., Gartside, S.: Proc. Chem. Soc. 413 (1960)

45. Koutecký, J., Čižek, J., Dubsky, J., Hlavaty, H.: Theoret. Chim. Acta (Berl.) 2, 462 (1964)

46. Koutecký, J., Hlavaty, K., Hochmann, P.: Theoret. Chim. Acta (Berl.) 3, 341 (1965)

47. Koutecký, J.: J. Chem. Phys. 47, 1501 (1967)

48. Hudson, B. S., Kohler, B. E.: Chem. Phys. Letters 14, 299 (1972); J. Chem. Phys. 59, 4984 (1973)

49. Christensen, R. L., Kohler, B. E.: Photochem. Photobiol. 18, 293 (1973)

50. Schulten, K., Karplus, M.: Chem. Phys. Letters 14, 305 (1972) 
51. Buenker, R. J., Whitten, J. L.: J. Chem. Phys. 49, 5381 (1968)

52. Dunning, T. H., Jr., Hosteny, R. P., Shavitt, I.: J. Am. Chem. Soc. 95, 5067 (1973)

53. Čižek, J., Paldus, J., Hubač, I.: Intern. J. Quantum Chem. 8, 951 (1974)

54. Paudus, J.: Intern. J. Quantum Chem. Symp. 8, 293 (1974)

55. Schulten, K., Ohmine, I., Karplus, M.: J. Chem. Phys. 64, 4422 (1976)

56. Paquette, L.: Z. Ang. Chemie 90, 114 (1978)

57. Giessner-Prettre, C., Pullman, A.: Theoret. Chim. Acta (Berl.) 17, 120 (1970); 13, 265 (1969); 18, 14 (1970); 20, 378 (1971)

58. Leclercq, J., Leclercq, J. M.: Chem. Phys. Letters 57, 54 (1978)

59. Spanget-Larsen, J., Gleiter, R.: Helv. Chim. Acta 61, 2999 (1978)

60. Dick, B.: Diplomarbeit, Univ. Köln 1977

61. McDiarmid, R.: J. Chem. Phys. 64, 514 (1976)

62. Mosher, O. A., Flicker, W. M., Kuppermann, A.: J. Chem. Phys. 59, 6502 (1973)

63. Buenker, R. J., Shih, S., Peyerimhoff, S. D.: Chem. Phys. Letters 44, 385 (1976)

64. Bender, C. F., Dunning, T. H., Jr., Schaefer, F. H, III, Goddard, W. A. III: Hunt, W. J.: Chem. Phys. Letters 15, 171 (1972); Ryan, J. A., Whitten, J. L.: Chem. Phys. Letters 15, 119 (1972); Basch, H.: Chem. Phys. Letters 19, 323 (1973)

65. Gavin, R. M., Rice, S. A.: J. Chem. Phys. 60, 3231 (1974)

66. Twarowski, A. J., Kliger, D. S.: Chem. Phys. Letters 50, 36 (1977)

67. Gavin, R. M., Riesemberg, S., Rice, S. A.: J. Chem. Phys. 58, 3160 (1973)

68. Karplus, M., Gavin, R. M., Rice, S. A.: J. Chem. Phys. 63, 5507 (1975)

69. Gavin, R. M., Jr., Weisman, Ch., McVey, J. K., Rice, S. A.: J. Chem. Phys. 68, 522 (1978)

70. Andrews, J. R., Hudson, B. S.: Chem. Phys. Letters 57, 600 (1978)

71. Platt, J. R.: J. Chem. Phys. 17, 481 (1949)

72. J. Chem. Phys. 23, 1997 (1955)

73. George, G. A., Morris, G. C.: J. Mol. Spectry. 26, 67 (1968)

74. Koch, E. E., Otto, A.: Chem. Phys. Letters 12, 476 (1972)

75. Mikami, N., Ito, I.: Chem. Phys. Letters 31, 472 (1977)

76. Ferguson, J., Reeves, L. W., Schneider, W. G.: Can. J. Chem. 35, 1117 (1957)

77. Koch, E. E., Otto, A., Radler, K.: Chem. Phys. Letters 21, 501 (1973)

78. Bergman, A., Jortner, J.: Chem. Phys. Letters 15, 309 (1972)

79. Kanda, Y., Shimada, R.: Spectrochim. Acta 15, 211 (1959)

80. Callomon, J. H., Dunn, T. M., Mills, I. M.: Phil. Trans. Roy. Soc. (London) A259, 499 (1966)

81. Koch, E. E., Otto, A.: Chem. Phys. Letters 12, 476 (1972)

82. Birks, J. B.: Chem. Phys. Letters 3, 567 (1969)

83. Twarowski, A. J., Kliger, D. S.: Chem. Phys. 20, 259 (1977)

84. Vaida, V., Robin, M. B., Kuebler, N. A.: Chem. Phys. Letters 58, 557 (1978)

85. DMS-UV Atlas of organic compounds. London: Butterworths 1971

86. Stachelek, T. M., Pazoha, T. A., McClain, W. M., Drucker, R. P.: J. Chem. Phys. 66, 4540 (1977)

Received May 30, 1979 